LA-UR-01-0888

Approved for public release; distribution is unlimited.

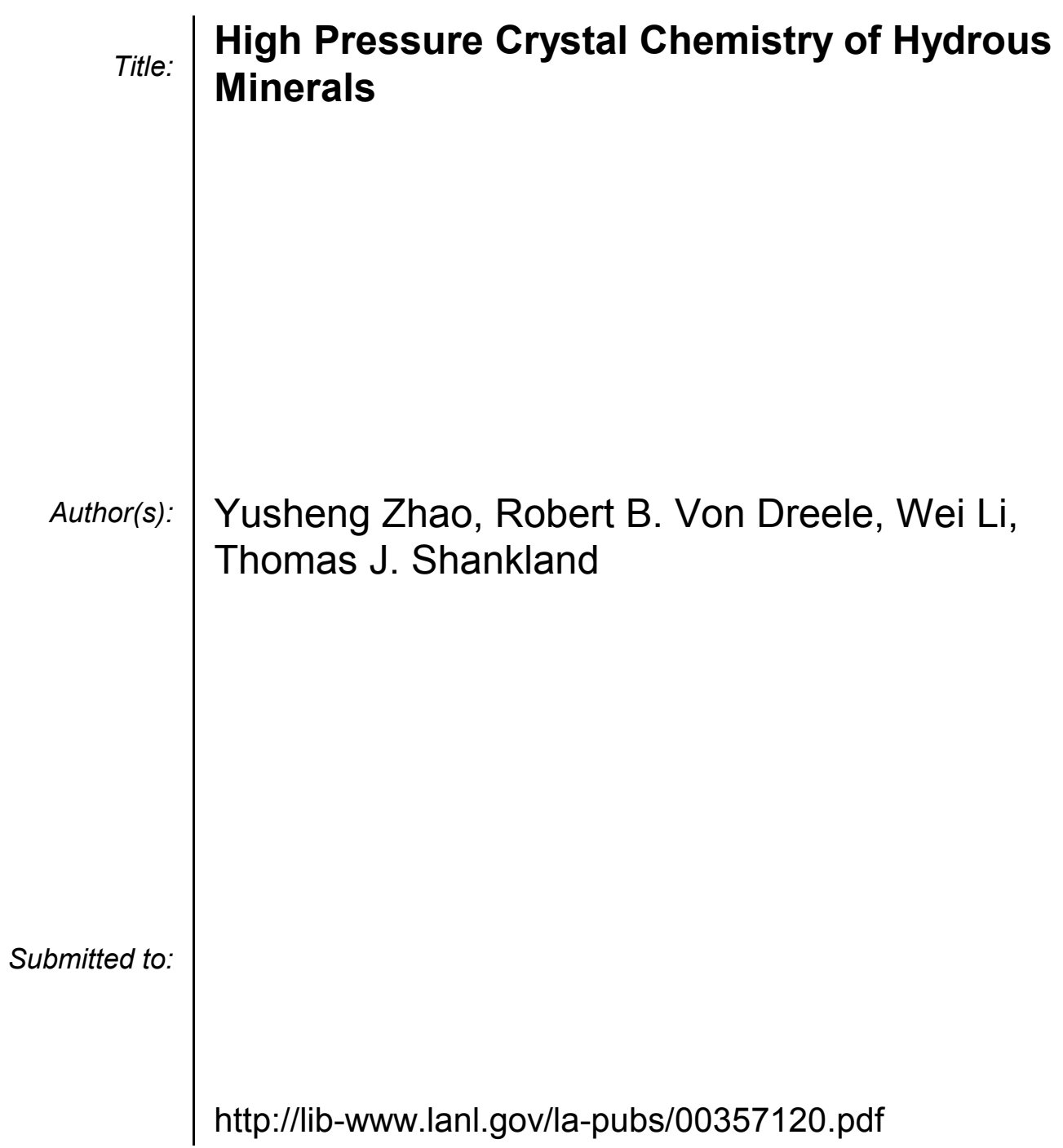

Los Alamos National Laboratory, an affirmative action/equal opportunity employer, is operated by the University of California for the U.S. Department of Energy under contract W-7405-ENG-36. By acceptance of this article, the publisher recognizes that the U.S. Government retains a nonexclusive, royaltyfree license to publish or reproduce the published form of this contribution, or to allow others to do so, for U.S. Government purposes. Los Alamos National Laboratory requests that the publisher identify this article as work performed under the auspices of the U.S. Department of Energy. Los Alamos National Laboratory strongly supports academic freedom and a researcher's right to publish; as an institution, however, the Laboratory does not endorse the viewpoint of a publication or guarantee its technical correctness. 


\title{
High Pressure Crystal Chemistry of Hydrous Minerals
}

\author{
Yusheng Zhao* \\ Robert B. Von Dreele, Wei Li, Thomas J. Shankland
}

\begin{abstract}
Hydrogen has long been appreciated for its role in geological processes of the Earth's crust. However, its role in Earth's deep interior has been neglected in most geophysical thinking. Yet it is now believed that most of our planet's hydrogen may be locked up in high pressure phases of hydrous silicate minerals within the Earth's mantle. This rocky interior (approximately 7/8 of Earth's volume) is conjectured to contain 1-2 orders of magnitude more water than the more obvious oceans (the "hydrosphere") and atmosphere. This project is aimed at using the capability of neutron scattering from hydrogen to study the crystal chemistry and stability of hydrogen-bearing minerals at high pressures and temperatures. At the most basic level this is a study of the atomic position and hydrogen bond itself. We have conducted experimental runs on hydrous minerals under high pressure and high temperature conditions. The crystallographic structure of hydrous minerals at extreme conditions and its structural stability, and hydrogen bond at high P-T conditions are the fundamental questions to be addressed. The behavior of the hydrous minerals in the deep interior of the Earth has been discussed.
\end{abstract}

\section{Background and Research Objectives}

The most common hydrous minerals found in Earth's upper mantle, the deep layer just beneath the crust, are phlogopite, amphibole, and serpentine. However, such minerals are unstable below depths of $\sim 200 \mathrm{~km}$. At greater depth, they decompose; it had been thought that all the water contained in their crystalline structure was released [Thompson, 1992]. In the last decade high-pressure and high-temperature experiments have demonstrated that hydrogen can be stored in crystals of nominally anhydrous minerals, such as olivine, pyroxene, and garnet [Bell \& Rossman, 1992]. In addition, the 
recent discovery of new high pressure hydrous phases has extended the existence of structurally incorporated hydrogen to depths much greater than $200 \mathrm{~km}$ where it is certain to have significant effects on evolution and dynamics of the mantle [Prewitt \& Finger, 1992]. Previous studies on high pressure hydrous phases have focused primarily on $\mathrm{OH}^{-}$ concentration and its effects on deformation, diffusion, and crystal defects. Only a few in-situ high pressure x-ray or neutron diffraction experiments have been conducted to look into the behavior of the hydrogen bond. (X-ray diffraction cannot accurately locate hydrogen atoms because of weak scattering of $\mathrm{x}$-rays from light elements.) There have been no reports of temperature effects on hydrogen bonds due to the tremendous experimental difficulties.

Even basic properties of the hydrogen bond at ambient conditions require study. Hydrogen (or, equivalently, water-) bearing crystalline systems are of fundamental interest not only in inorganic chemistry but also because of hydrogen bonding in biological systems (e.g., in $\mathrm{H}_{2} \mathrm{O}$ and $\mathrm{CH}_{4}$ ). Increasing pressure and temperature change inter-atomic distances and offer a powerful new means of elucidating forces associated with hydrogen bonding, thus providing significant constraints on molecular-dynamic and other atomistic modeling. Also, such experiments are now motivated by the recent discovery that pressure-induced phase transformations can involve just the hydrogen sublattice of crystalline oxides.

Neutron diffraction yields unique information on crystallographic sites, inter-atomic distances, and thermal vibration in hydrogen-bonded systems [Pynn, 1990, Von Dreele, 1990]. Systematic study using high P-T neutron diffraction to investigate the structural and thermal parameters of hydrous magnesium silicates helps the understanding of the role water plays in deep geophysical process. The focus of the study is on the bond length, bond angle, and thermal vibration parameters of hydrogen atoms. Thus, both stability and breakdown mechanisms of the hydrous phases can be inferred at the atomic scale. 


\section{Importance to LANL's Science and Technology Base and National R\&D Needs}

At Los Alamos, we recently extended neutron diffraction experiments to simultaneous high pressure and temperature of $10 \mathrm{GPa}$ and $1500 \mathrm{~K}$ using LANSCE/HIPD beamline. This breakthrough of in-situ high P-T neutron diffraction is especially important to the study of hydrous minerals because all other diffraction and spectroscopic techniques are unable to locate hydrogen positions. With the recent development in high P-T in-situ neutron diffraction at LANSCE, we opened a new window for examining the scientific problems associated with hydrous minerals. The proposed study of crystal chemistry of hydrous magnesium silicates provides important information on the transformation of $\mathrm{Si}$ coordination from four to six with increasing P-T and on structural features that permit hydrogen to be retained under extreme conditions. Studies of hydrogen bonds at high pressures and temperatures have significant implications not only to planetary and Earth sciences but also to materials sciences and energy research. Because of their potential as new means of energy storage and transportation, hydrides have been important in materials science. The high energy density, low pollution, and vast resources of hydrogen have attracted active research for decades. Effects of pressure, one of the most important thermodynamic variables, have not been adequately explored and are thus prime objects for neutron diffraction studies. This study of hydrogen bond in crystalline structures at high pressures and high temperatures will definitely shed light on the subject.

\section{Scientific Approach and Accomplishments}

We perform the neutron diffraction experiments with a unique high P-T cell assembly specially developed at Los Alamos for the measurements. The ceramic gasket and carbon furnace cell assembly will be placed in a Paris-Edinburgh (P-E) toroidal press [Besson et al., 1995]. The ceramic gasket is chosen for strength and thermal/electric insulation. High temperature is achieved via internal resistance heating of a cylindrical carbon furnace, which ensures stability and homogeneity of the temperature distribution. Heating is by a controlled DC power supply, and temperature is read out from dual thermocouples placed immediately next to the sample. Pressure will be calibrated using 
the Decker equation of state for $\mathrm{NaCl} / \mathrm{CsCl}$ [Decker, 1971] and the high-T BirchMurnaghan equation of state for MgO [Birch, 1981, Saxena \& Zhang, 1990]. The "warm-up" of $700 \sim 800 \mathrm{~K}$ in temperature will ensure hydrostatic conditions on the mixture of sample material and pressure calibrants.

Because hydrogen incoherently scatters neutrons, highly hydrous samples should be deuterated to enhance data collection rates. We have collaborated with scientists at University at Stony Brook (J. B. Parise and Jianzhong Zhang), Arizona State University (K. Leinenweber), and University of Louisville (G. A. Lager) to synthesize and characterize deuterated samples such as: brucite $\operatorname{Mg}(\mathrm{OD})_{2}$, hydrous-A phase $\mathrm{Mg}_{7} \mathrm{Si}_{2} \mathrm{O}_{8}(\mathrm{OD})_{6}$, and hydrogarnet $\mathrm{Ca}_{3} \mathrm{Al}_{4}\left(\mathrm{O}_{4} \mathrm{D}_{4}\right)_{3}$ etc. The crystal structure and characteristics of hydrogen bonds have been studied for these samples at ambient and high-P and high-T conditions. Broadening of diffraction peaks in some high-P and room$\mathrm{T}$ experiments had hampered refinement of structural and thermal parameters. Preliminary "warm-up" dramatic increases the sharpness and distinctiveness of diffraction patterns as specimens anneal with temperature. The results show that diffraction quality at high pressures generally improves with temperature.

We have successfully synthesized several deuterated hydrous minerals including brucite (MgOD, @ 300 $\mathrm{C}$, 12 hours), talc $\left(\mathrm{Mg}_{3} \mathrm{Si}_{4} \mathrm{O}_{10}(\mathrm{OD})_{2}\right.$, @ 2.5 kbar, $600^{\circ} \mathrm{C}, 48$ hours) and the $10-\AA$ phase $\left(\mathrm{Mg}_{3} \mathrm{Si}_{4} \mathrm{O}_{14} \mathrm{D}_{6}, @ 70\right.$ kbar, $450^{\circ} \mathrm{C}, 10 \sim 48$ hours $)$. The deuterated samples have been analyzed by powder x-ray diffraction, micro Raman spectroscopy, and Fourier transformed IR absorption scpectroscopy. Our proposal has obtained the highest rank for all BES general user proposals of HIPD beam line including both DP and BES programs for LANSCE beam time.

Neutron diffraction experiment has been conducted on deuterated hydrous mineral, brucite $\mathrm{Mg}(\mathrm{OD})_{2}$. Neutron diffraction is sensitive to the hydrogen (deuterium) position which allow us to study the details of the crystal structure of hydrous minerals. The crystal structure of the deuterated sample was refined using Rietveld technique and the atomic position and thermal vibrations were determined accordingly. The refinement results were compared with previous experimental results and got very good agreement. 
Plotted in Figure 1 are two sets of crystal structures with different crystallographic models for brucite. The top pictures show the traditional view of brucite that the hydrogen atoms are positioned at the $(2 d)$ site with a full occupation of 1.0 , while the bottom pictures show the new prospect of hydrogen occupation of $1 / 3$ at the $(6 i)$ site. It is a quite challenging study to distinguish these two crystallographic models experimentally.

The high-resolution time-of-flight neutron diffraction pattern we collected at LANSCE for the brucite sample covers a wide range of $d$-spacing from $0.4 \AA$ to $4.0 \AA$, which make the accurate determination of the crystal structure possible. Shown in Figure 2 are neutron diffraction patterns in observed and calculated forms. The structural models are tabled in the right-side diagram and their differences are listed at the top and the bottom, respectively. The left-top diagram is the refinement result for the traditional model of full occupation of hydrogen atoms at $(2 d)$ site and the left-bottom one is the new prospect with $1 / 3$ occupation probability for the hydrogen been sited at (6i) position.

Thermal vibration of different atoms are refined and listed in Figure 2. For both models of the deuterated brucite $\mathrm{Mg}(\mathrm{OD})_{2}$, the $\mathrm{Mg}$ atom does not vibrate much as indicated by a small $U_{\text {iso }}$ value, while the oxygen atom has a median $U_{\text {iso }}$ value and the deterium atom has the highest $U_{\text {iso }}$ value. We believe that in case of $\operatorname{Mg}(\mathrm{OH})_{2}$ the hydrogen atom may have a much higher thermal vibration than the deuterium atom because the mass-difference between the two. One significant difference between two models is that the observed thermal vibration is $\mathrm{U}_{\text {iso }}=2.923 \times 10^{-2} \AA^{2}$ for the (2d) site, a rather large value for room temperature measurement; while $\mathrm{U}_{\mathrm{iso}}=1.845 \times 10^{-2} \AA^{2}$ observed for the (6i) site is a more realistic measure. The observation of thermal motion of hydrogen atoms plus the goodness of the fit (as indicated by $\chi^{2}$ ) suggest that the new prospect is a better structural model for the brucite.

We have also worked on the deuterated hydrous mineral Phase- $\mathrm{A} \mathrm{Mg}_{7}{ }^{\mathrm{IV}} \mathrm{Si}_{2} \mathrm{O}_{8}(\mathrm{OD})_{6}$ synthesized at high pressure and temperature conditions of $\mathrm{P}=10 \mathrm{GPa}$ and $\mathrm{T}=1000 \mathrm{C}$, with the mixture of three (3) parts of brucite MgOD (deuterited sample) and two (2) parts of forsterite $\mathrm{Mg} 2 \mathrm{SiO} 4$ (end-member olivine). The time-of-flight neutron diffraction data 
were collected at LANSCE/HIPD with high resolution ( $151^{\circ}$ bank) and moderate resolution $\left(90^{\circ}\right.$ bank), respectively. Shown in Figure 3 are neutron diffraction patterns in observed, calculated, and difference forms. The crystal structure of the deuterated sample was refined using Rietveld technique and the atomic position and thermal vibrations were determined accordingly. With the wide range of $d$-spacing from $0.5 \AA$ to $3.0 \AA$, we can make accurate determination of the crystal structure. The refinement results were compared with previous experimental results by Kurt Lenerweber (ASU) and John Parise (USB) and got very good agreement. Neutron diffraction is sensitive to the hydrogen (deuterium) positions that allow us to study the details of the crystal structure of hydrous minerals (Figure. 4). The in-situ high pressure/temperature neutron diffraction experiment will reveal the structure response to the P-T conditions and the details of the dehydration process.

\section{Publications}

Zhao, Y., F. Chu, R. B. Von Dreele, Q. Zhu, D. E. Cox, (2000), Structural Phase Transitions of $\mathrm{HfV}_{2}$ at Low Temperatures, Acta Crystallography B, 56, 601-606.

Zhao, Y., A. C. Lawson, J. Zhang, B. I. Bennett, R. B. Von Dreele, (2000), Thermoelastic Equation of State of Molybdenum, Physical Review B, 62, 8766-8776.

Zerda, T. W., W.Xu, A. Zerda, Y. Zhao, R. B. Von Dreele, (2000), High Pressure Raman and Neutron Scattering Study on Structure of Carbon Black Particles, Carbon, 38 $355-361$.

Zhao, Y., R. B. Von Dreele, J.G. Morgan, (1999), A High P-T Cell Assembly for Neutron Diffraction up to $10 \mathrm{GPa}$ and $1500 \mathrm{~K}$, High Pressure Research, 16, 161-177.

Zhao, Y., R. B. Von Dreele, D. J. Weidner, (1999), Correction of Diffraction Optics and P-V-T Determination Using Thermoelastic Equations of State of Multiple Phases, Journal of Applied Crystallography, 32, 218-225.

Zerda, T. W., A. Zerda, Y. Zhao, R. B. Von Dreele, (1999), Structure of Carbon Black Particles, High Pressure Molecular Science, eds. R. Winter and J. Jonas, 225-230.

Marasinghe, G.K., W.J. James, P.C. Ezekwenna, R. Luo, W.B. Yelon, Y. Zhao, R.B. Von Dreele, M. Ellouze, and Ph. I'Heritier, (1998), Pressure Induced Reversal of The 
Volume Expansion Caused by Interstitial Nitrogen in $\mathrm{Nd}_{2} \mathrm{Fe}_{17} \mathrm{~N}_{3}$, Journal of Apply Physics, 83, 6911-6916.

\section{References}

Bell, D.R., and G.R. Rossman, Water in Earth's mantle: the role of nominally anhydrous minerals, Science, 255, 1391-1397 (1992).

Besson, J.M., S. Klotz, G. Hamel, I. Makarenko, R.J. Nelmes, J.S. Loveday, R.M. Wilson, and W.G. Marshall, High pressure neutron diffraction, present and future possibilities using the Paris-Edinburgh cell, High Pressure Research, 14, 1-6 (1995).

Birch, F., Finite elastic strain of cubic crystal, Physical Review, 71, 809-824 (1947).

Birch, F., Composition of the Earth's mantle and core, Geophys. Res. Lett., 4, 295-311 (1961).

Decker, D. L., High-pressure equation of state for $\mathrm{NaCl}, \mathrm{KCl}$, and $\mathrm{CsCl}$, J. Appl. Phys. 42, 3239-3244 (1971).

Prewitt, C.T., and L. W. Finger, Crystal chemistry of high-pressure hydrous magnesium silicates, High Pressure Research: Application to Earth and Planetary Sciences, eds. Y. Syono \& M.H. Manghnani, 269-274 (1992).

Pynn, R., Neutron scattering — a primer, Los Alamos Science, 19, 1-31 (1990).

Saxena, S.K., and J. Zhang, Thermochemical and pressure-volume-temperature systematics of data on solids, examples: tungsten and MgO, Phys Chem. Minerals, 17, 45-51 (1990).

Thompson, A.B., Water in the Earth's upper mantle, Nature, 358, 295-302 (1992).

Von Dreele, R.B., X-ray and neutron crystallography - a powerful combination, Los Alamos Science, 19, 132-157 (1990).

\section{Figures (next pages)}



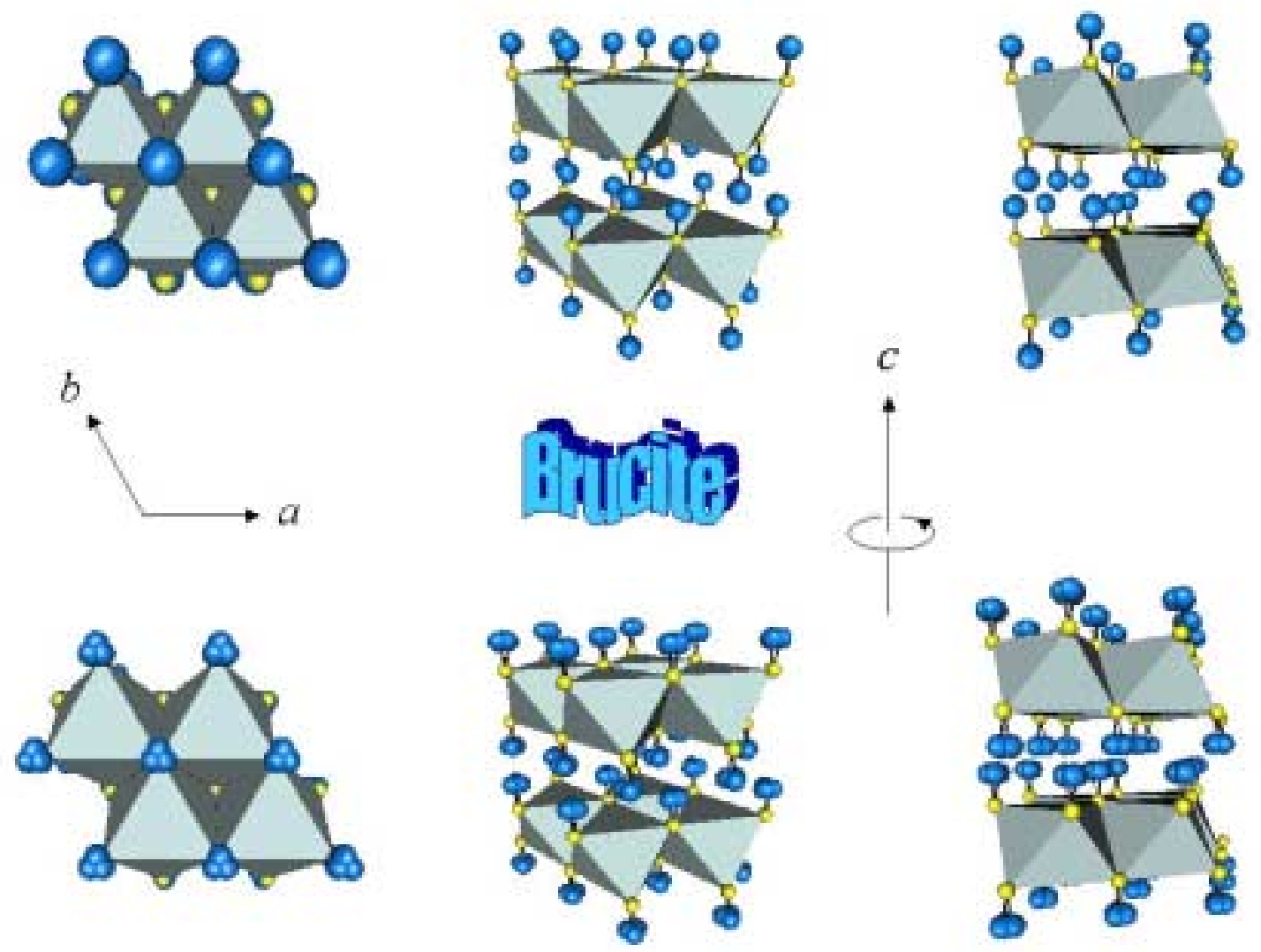

Figure 1, Structural models for brucite $\mathrm{Mg}(\mathrm{OH})_{2}, \mathrm{MgO}_{6}$ octahedral has shared edges and the hydrogen is the tangling atom (the small blue balls). The top pictures show the traditional view of brucite that the hydrogen atoms are positioned at the $(2 d)$ site with a full occupation of 1.0, while the bottom pictures show the new prospect of hydrogen occupation of $1 / 3$ at the $(6 i)$ site. 

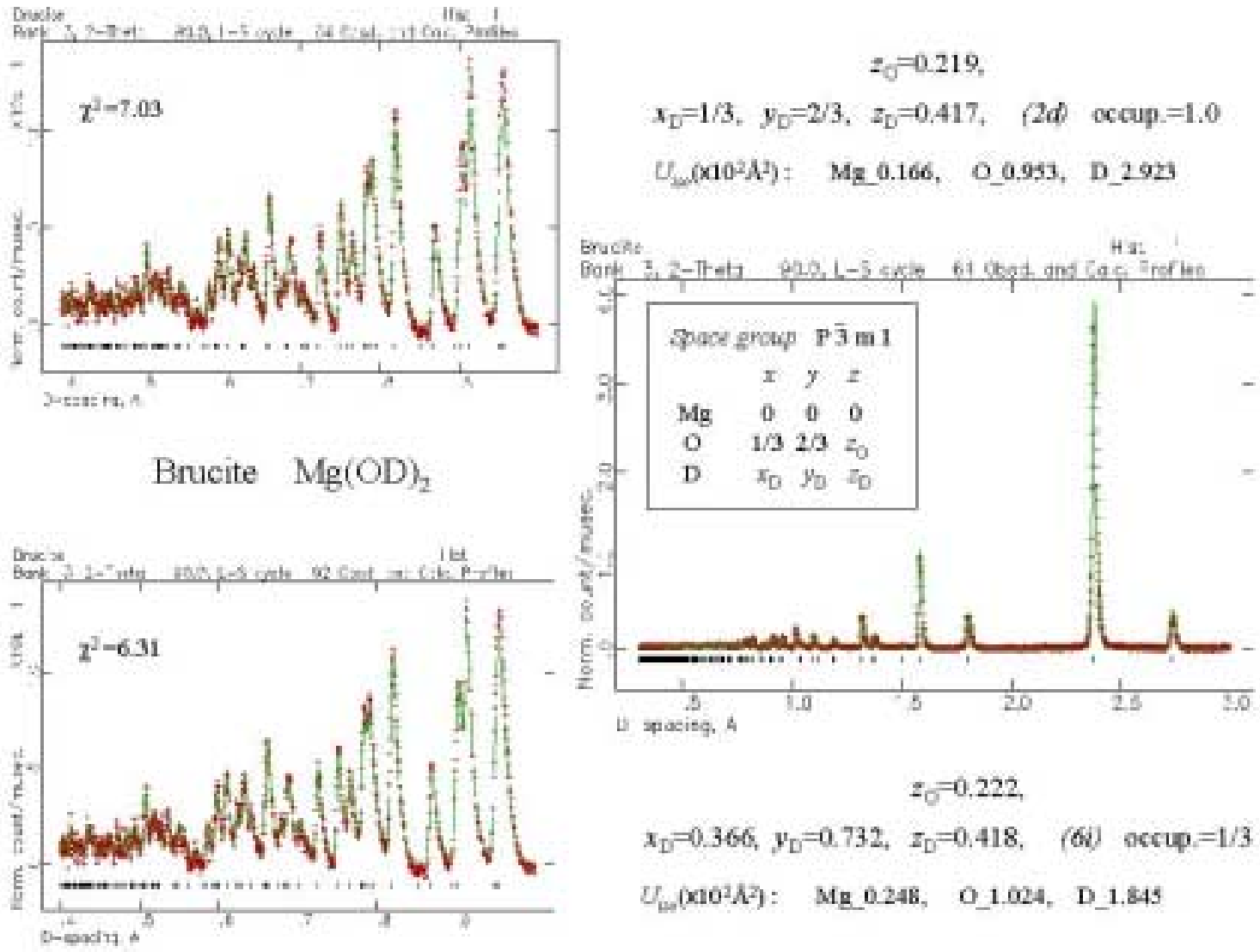

Figure 2, Structural refinement results of neutron diffraction of $\operatorname{Mg}(\mathrm{OD})_{2}$. The right-side shows the overall diffraction pattern and the left-side shows detailed spectra at small $d$-spacing. The peak positions of the diffraction pattern reveal the information about lattice dimensions, while, the peak intensities (especially those at small $d$-spacing) can be used to refine atomic positions and thermal vibrations. 


\section{Hydrous Mineral Phase_A $\mathrm{Mg}_{7} \mathrm{Si}_{2} \mathrm{O}_{8}(\mathrm{OD})_{6}$}
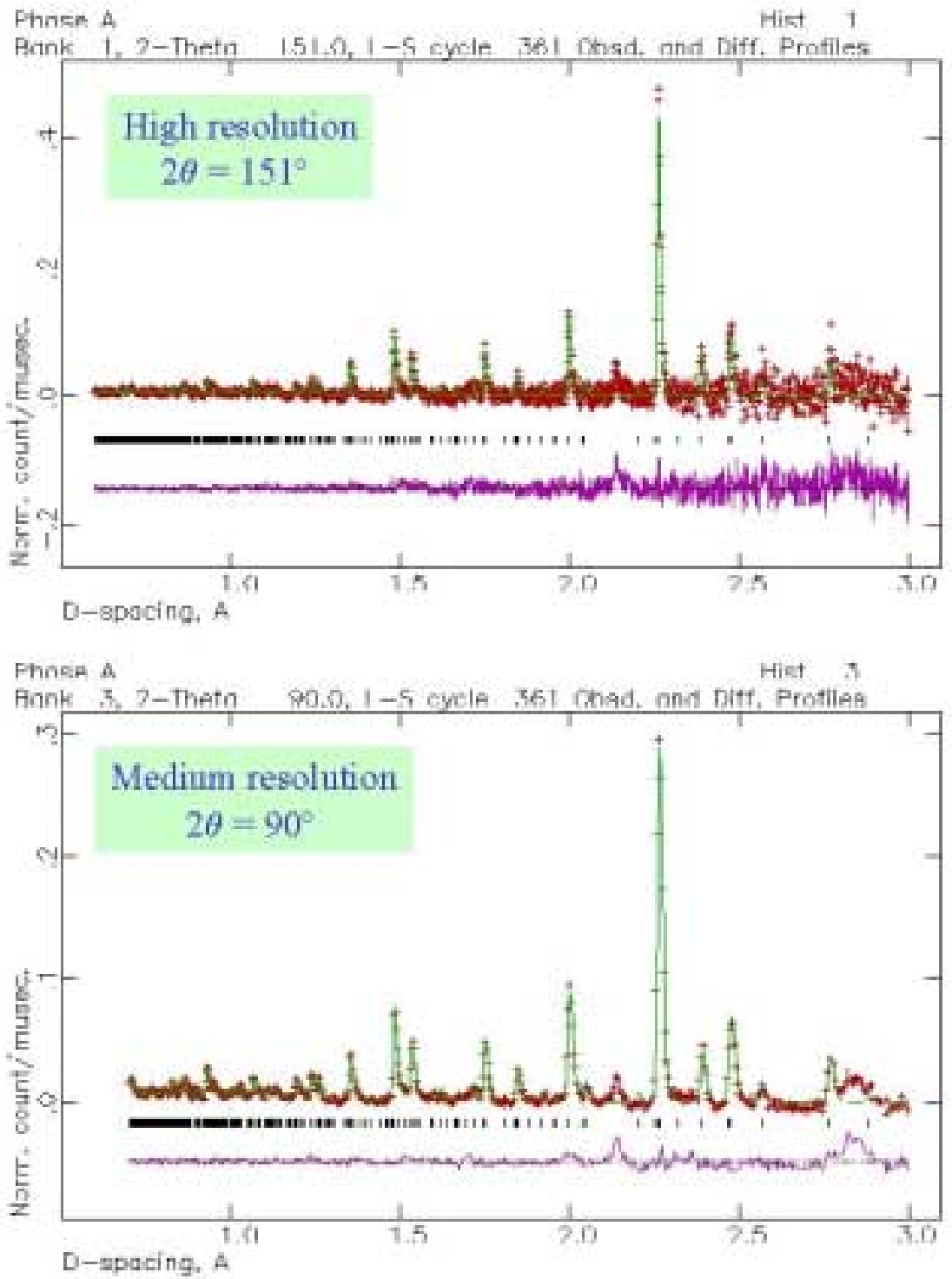

Figure 3, The Rietveld refinement for the time-of-flight powder neutron diffraction pattern of deuterited phase- $\mathrm{A} \mathrm{Mg}{ }_{7}{ }^{\mathrm{IV}} \mathrm{Si}_{2} \mathrm{O}_{8}(\mathrm{OD})_{6}$. Observed data are indicated by a cross "+"; and the calculated pattern is drawn as a continuous red line; and the difference between calculated and observed patterns are shown at the bottom. The Bragg reflection positions are shown by short vertical bars marked below the diffraction pattern. 


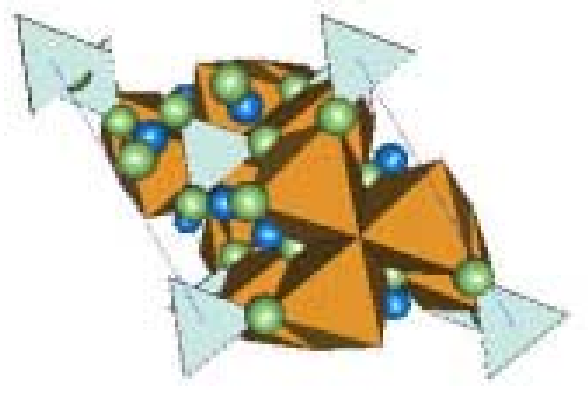

\section{Hydrous Mineral \\ Phase A \\ $\mathrm{Mg}_{7} \mathrm{Si}_{2} \mathrm{O}_{8}(\mathrm{OD})_{6}$}
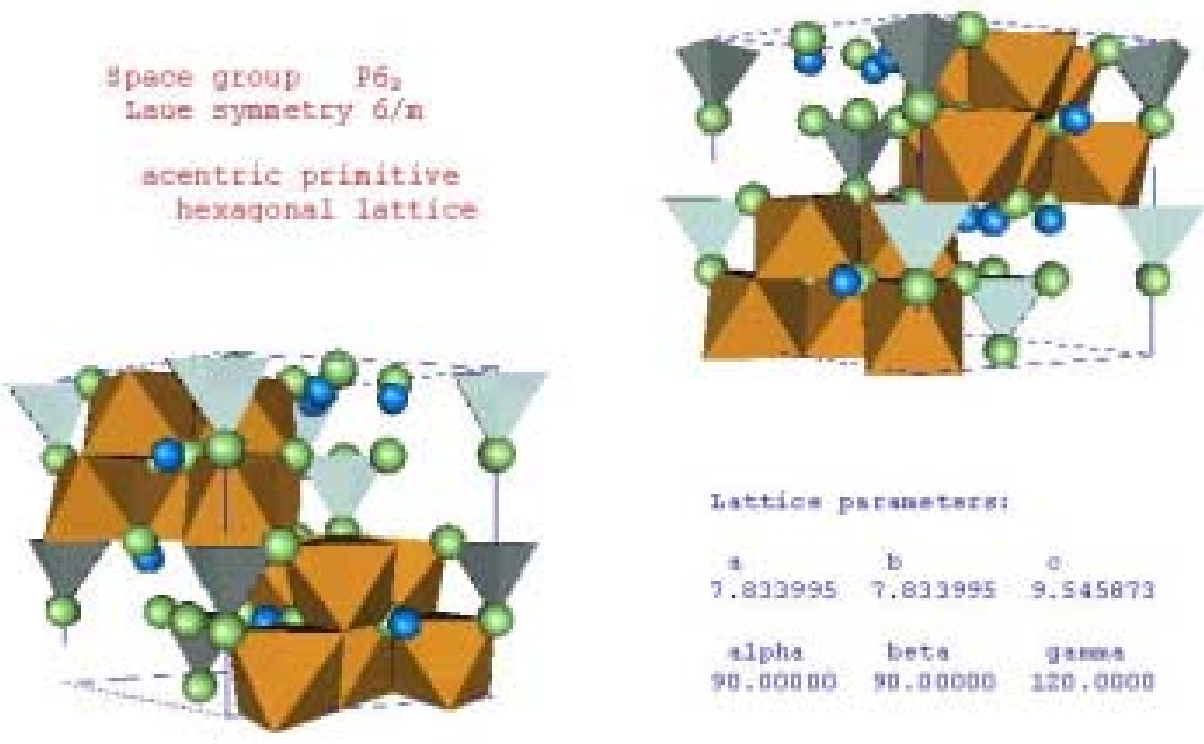

\begin{tabular}{|c|c|c|c|}
\hline Atan & $\mathrm{z}$ & $\gamma$ & $\mathrm{z}$ \\
\hline$m=$ & .2750 & -4524 & .3761 \\
\hline no & , t2128日 & .2422 & .1042 \\
\hline mo &, 3323 & .6667 & -1214 \\
\hline 31 & . 5667 & .3333 & .1705 \\
\hline 91 & .0000 & .0000 & .3922 \\
\hline 0 & +2525 & .0344 & $-.025 \mathrm{a}$ \\
\hline 0 & +4697 & , 0 a 194 & -4602 \\
\hline 0 & 4523 & .2329 & .7395 \\
\hline 0 & .1601 & .4452 & .0361 \\
\hline 0 & .6667 & ב3.393. & .0039 \\
\hline 0 &, 0000 & .0000 & .2417 \\
\hline D & .0387 & .3664 & .2368 \\
\hline D & 4 1672 & 7106 & .9235 \\
\hline
\end{tabular}

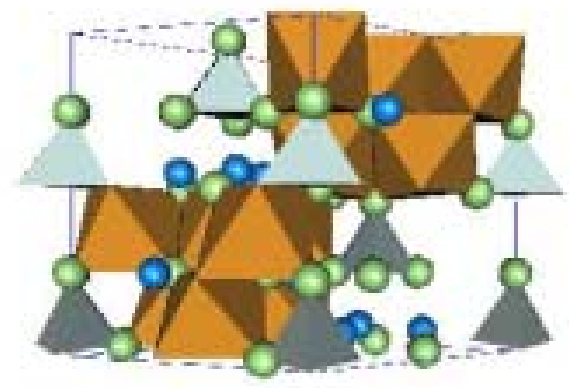

Figure 4, Crystal structure of Phase- $\mathrm{A} \mathrm{Mg}_{7}{ }^{\mathrm{V}} \mathrm{Si}_{2} \mathrm{O}_{8}(\mathrm{OD})_{6}$ shown from different viewangles. The $\mathrm{Mg}$-centered octahedrals and the Si-centered tetrahedrals are the major building blocks of the structure and the hydrogen (blue ball, deuterium in the current case) atoms are tangled with the oxygen atoms (green ball). There are quite large void 
spaces in the crystal structure which is expected to collapse at higher pressures. The refined lattice parameters and structure parameters are listed on the diagrams. 